(6) Bundesamt für Industrie, Gewerbe und Arbeit: Beschäftigung und Arbeitsmarkt im Jahre 1981. In: Die Volkswirtschaft H. 3, März 1982, 119-122.

(7) CANDINAS, R. (1982): Direkte Auswirkungen von Industrieanlagen und Hotelbetrieben auf die Beschäftigungsstruktur Eine empirische Untersuchung in der Gemeinde Disentis, Diplomarbeit am Geographischen Institut der Universität Zürich, Baden, 84-88.

(8) Als Beispiele sind zu erwähnen: BELLWALD, A. u. a. (1981): Die wirtschaftliche Bedeutung des Tourismus im Wallis (Hrsg.: Volkswirtschaftsdepartement des Kantons Wallis, Amt für Tourismus), Brig.

BEZZOLA, A. (1975): Probleme der Eignung und Aufnahmekapazität touristischer Bergregionen, St.Galler Beiträge zum Fremdenverkehr und zur Verkehrswirtschaft, Reihe Fremdenverkehr Bd. 7, Bern, 110-113.

FLURY, M. (1981): Kantonale Entwicklungsplanung - Die wirtschaftliche Bedeutung des Fremdenverkehrs in Appenzell A.R. In: Konjunktur - Analysen und Prognosen, Monatsbericht, Institut für Wirtschaftsforschung ETH Zürich Nr. 2, 20-44.

SCHMIDHAUSER, H.P. (1978): Der Beschäftigungseffekt des Fremdenverkehrs im tertiären Sektor - dargestellt am Beispiel der Schweiz. In: Ender Walter A. (Hrsg.): Beiträge zur Fremdenverkehrsforschung (Festschrift zum 70. Geburtstag von Prof. Paul Bernecker), Institut für Fremdenverkehrsforschung der Wirtschaftsuniversität Wien, 51-57.
(9) Beratende Kommission für Fremdenverkehr des Bundesrates (1979): Das Schweizerische Tourismuskonzept. Grundlagen für die Tourismuspolitik, Schlußbericht, Bern, 55.

(10) Die wirtschaftliche Bedeutung des Tourismus im Wallis, Tab. 66, 128.

(11) Zur Aussagefähigkeit des Intensitätsbegriffes vgl. auch Österreichisches Institut für Raumplanung (1980): Zur Problematik des einsaisonalen Fremdenverkehrs in bezug auf Raumordnung und Wirtschaftsentwicklung, Wien, 24 und 43.

(12) KELLER, Th. (1983): Tourismus und Berggebietsförderung - Eine empirische Untersuchung über die Auswirkungen von Investitionen in die touristische Infrastruktur in alpinen Fremdenverkehrsorten der Schweiz, Dissertation Universität Zürich, Zürich.

(13) Zum Problem der Vermietung von Ferienwohnungen durch Einheimische vgl. Die wirtschaftliche Bedeutung des Tourismus im Wallis, 34-40.

DARBELLAY, Ch. (1982): Le tourisme rural, élément d'une politique de développement régional. In: VPK Nr. 5, 153-155.

CASAULTA, P. (1982): Bedeutung und Probleme der einheimischen Parahotellerie - Am Beispiel Disentis-Mustèr/GR, Diplomarbeit am Geographischen Institut der Universität Zürich.

(14) Vgl. dazu TSCHIDERER, F. (1980): Ferienortsplanung Eine Anwendung unternehmensorientierter Planungsmethodik auf den Ferienort, St. Galler Beiträge zum Fremdenverkehr und zur Verkehrswirtschaft, Reihe Fremdenverkehr Bd.12, Bern.

\title{
Bundesbehörden und Tourismus
}

\section{Das tourismuspolitische Instrumentarium}

Der Bund ist aufgrund mittelbarer Verfassungskompetenzen für touristische Angelegenheiten zuständig. Es bestehen mehrere Erlasse, die im finanziellen und regulativen Bereich direkt dem Tourismus zugerechnet werden können. Der Bund leistet so jährlich über 60 Mio. Franken für die Förderung des Tourismus (ohne touristischen Verkehr). Darunter fällt beispielsweise die Finanzierung der touristischen Landeswerbung, die 1983 voraussichtlich über 22 Mio. Franken ausmachen wird. Das wichtigste tourismuspolitische Steuerungsinstrument liegt im Bereich der touristischen Transportanlagen. Konzessionen und Bewilligungen von Luftseilbahnen und Skiliften lösen bei Realisierung der Projekte zahlreiche Investitionen im Siedlungs- und Verkehrsbereich aus. Für den Tourismus von Bedeutung sind zudem die Maßnahmen des Bundes im Bereich seiner Rahmenbedingungen, insbesondere der Infrastruktur, des Verkehrs, des
Arbeitsmarktes und der Berufsbildung, der Außenwirtschaft, aber auch der Raumplanung und des Landschaftsschutzes.

\section{Tourismus als Teilbereich der Regierungspolitik}

Auf Bundesebene befassen sich Parlament, Regierung und Verwaltung mit Geschäften, die den Tourismus betreffen. Gegen 120 Parlamentarier sind Mitglieder der Parlamentarischen Gruppe für Tourismus. Es handelt sich dabei um eine informelle Vereinigung eines weiten Kreises Interessierter und nicht um eine touristische Lobby. Zuständig für die Tourismuspolitik ist der Bundesrat. Er verabschiedete im Frühjahr 1981 das Schweizerische Tourismuskonzept, welches als tourismuspolitische Leitlinie in den Richtlinien für die Regierungspolitik der laufenden Legislaturperiode enthalten ist. Dem Bundesrat steht eine beratende Kommission für Fremdenverkehr bei, die für ihn 
tourismuspolitische Geschäfte begutachtet. Der Kommission gehören je zwei Vertreter aus den Bereichen Forschung, Politik und Praxis an. Zudem sind die beiden öffentlich-rechtlichen Körperschaften des Bundes im Bereiche des Tourismus, die Schweizerische Verkehrszentrale und die Schweizerische Gesellschaft für Hotelkredit sowie fünf eidgenössische Departemente in der Kommission vertreten.

\section{Der Fremdenverkehrsdienst als Fachstelle fuir Tourismus der Bundesverwaltung}

Auf Verwaltungsebene besteht mit dem Fremdenverkehrsdienst des Bundesamtes für Verkehr eine Fachstelle für Tourismus, die grundsätzlich alle den Tourismus betreffenden Angelegenheiten bearbeitet. Ausnahme bilden die ausdrücklich durch Gesetz und Verordnung einer anderen Stelle zugeordneten Aufgaben (z. B. Fremdenverkehrsstatistik). Der Fremdenverkehrsdienst setzt sich für günstige Rahmenbedingungen und eine geordnete Entwicklung des Tourismus als bedeutende Freizeitform und Wirtschaftsbranche ein. Obwohl personell bescheiden dotiert, versucht er, die Anliegen des Schweizer Tourismuskonzeptes in die Praxis umzusetzen. Der Bundesrat hat dazu mit der Verbindlicherklärung des Konzeptes für die Verwaltung die Voraussetzungen geschaffen. Als wesentliches Instrument für die Durchsetzung der Tourismuspolitik kann der Fremdenverkehrsdienst die Konzessionspolitik für touristische Transportanlagen einsetzen. Im Bereiche des touristischen Marketings beschäftigt sich der Dienst mit der Aufsicht und der Finanzierung der Landeswerbung der SVZ und unterstützt die Informations- und Beratungstätigkeit des Schweizerischen Fremdenverkehrsverbandes zugunsten der landesweiten Angebotsgestaltung. Zudem befaßt er sich mit zahlreichen tourismuspolitischen Sondergeschäften, die von der Aufsicht über ein Feriendorf für Jugendliche bis zur Bearbeitung von Beitragsgesuchen für die Durchführung Olympischer Spiele reichen. Der Fremdenverkehrsdienst vertritt die Schweiz auch in den intergouvernementalen Organisationen, beispielsweise in der Weltorganisation für Tourismus oder im Tourismuskomitee der OECD, und arbeitet bilateral mit den Tourismusbehörden anderer Reiseländer zusammen.

Ein besonderes Anliegen der Fachstelle ist die Koordination der touristischen Anliegen auf Bundesebene und die Zusammenarbeit mit den Kantonen und Trägern des Tourismus. Der Fremdenverkehrsdienst führt das Sekretariat der beratenden Kommission für Fremdenverkehr. Er arbeitet in den Organen nationaler touristischer Institutionen mit. Tourismus ist eine Querschnittaufgabe, die in viele Lebensbereiche hineingreift. Bei der komplexen und dezentralisierten Organisation des schweizerischen Tourismus braucht es stetige Bemühungen, da $\beta$ der Blick auf das touristische Ganze nicht verlorengeht. Zusammen mit unseren Partnern bei Behörden und Privaten setzen wir uns dafür ein. Ab 1984 wird der Fremdenverkehrsdienst dem Bundesamt für Industrie, Gewerbe und Arbeit im Volkswirtschaftsdepartement angegliedert.

Fremdenverkehrsdienst, Bundesamt für Verkehr, Bundeshaus Nord, CH-3003 Bern, Tel. 031/6157 94/92

Leiter: Dr. Peter Keller, Stv.: lic. rer. pol. Peter Schmid

\section{Die Sektion Tourismus im Bundesamt für Statistik}

Das Bundesamt für Statistik (BFS) wurde 1933 beauftragt, eine «regelmäßige Statistik der in den Beherbergungsstätten abgestiegenen Gäste» durchzuführen. Die Betreuung der fremdenverkehrsstatistischen Erhebungen obliegt der Sektion Tourismus, die 26 Mitarbeiterinnen und Mitarbeiter beschäftigt.

Die gesetzlichen Grundlagen zur eidgenössischen Fremdenverkehrsstatistik aus dem Jahre 1933 wurden vorerst nur teilweise ausgenützt; sie verlangten vorderhand lediglich die Erfassung der in den Hotel- und Kurbetrieben abgestiegenen Gäste. Heute werden diese Grunddaten mittels offizieller Formulare direkt bei den Inhabern und Leitern der rund 8000 meldepflichtigen Hotels, Pensionen, Gasthäuser, Motelș, Bäder- kliniken, Volksheilbäder, Kurhäuser und Höhensanatorien eingeholt. Ankünfte und Logiernächte aufgeteilt nach Wohnsitzländern der Gäste - werden dabei monatlich registriert, ebenso das Bettenangebot, die Öffnungszeiten der Betriebe und die Zahl der beschäftigten Personen. Jeweils am 25. des dem Berichtsmonat folgenden Monats sind die ersten gesamtschweizerischen Ergebnisse verfügbar, die in Form einer Pressemitteilung verbreitet werden. Die DetailÜbersichten erscheinen etwa zehn Tage später. Publiziert und kommentiert werden Monats-, Saisonund Jahresergebnisse, geordnet nach touristischen Regionen, Zonen, Fremdenverkehrsorten, Städten, Herkunftsländern, Preisklassen, Betriebsarten usw. 